\title{
Perturbation theory and renormalization group equations
}

\author{
Daniel F. Litim* \\ Theory Division, CERN, CH-1211 Geneva 23, Switzerland \\ Jan M. Pawlowski ${ }^{\dagger}$ \\ Institut für Theoretische Physik III, Universität Erlangen, D-91054 Erlangen, Germany
}

(Received 22 November 2001; published 5 April 2002)

\begin{abstract}
We discuss the perturbative expansion of several one loop improved renormalization group equations. It is shown that in general the integrated renormalization group flows fail to reproduce perturbation theory beyond one loop.
\end{abstract}

DOI: 10.1103/PhysRevD.65.081701

PACS number(s): 11.10.Gh, 05.10.Cc

\section{INTRODUCTION}

Renormalization group (RG) methods are an essential ingredient in the study of nonperturbative problems in continuum and lattice formulations of quantum field theory. A number of RG equations have been proposed, where the starting point is the (infrared) regularized one-loop effective action. Taking the derivative with respect to the infrared scale together with a subsequent one-loop improvement leads to a flow for the effective action. The merit of such an equation is its flexibility, as it allows for nonperturbative approximations not bound to the weak coupling regime. Thus, these flows are particularly interesting for theories where one has to resort to truncations because the full problem is too hard to attack. Indeed, surprisingly good results concerning critical exponents in scalar theories have been obtained within simple approximations to a particular version of a one-loop improved RG [1], based on a proper-time representation of the one-loop effective action [2]. It has also been suggested that the proper-time RG may be an interesting tool for gauge theories, since the regularization respects a local non-Abelian gauge symmetry [3].

However, results obtained within a truncated system are only as good as the accompanying quality checks. Apart from the inherent problems of these checks, the present situation requires additional care, since most of the one-loop improved RG flows lack a first-principles derivation. Such flows suffer from a severe conceptual problem. It is unclear whether they are only approximations to flows for the full effective action or whether they represent an exact flow. The latter is indeed known to hold true for exact RG (ERG) flows $[4,5]$ (for reviews see [6]). They can be obtained within a one-loop improvement, but also from a first-principles derivation, mostly done within a path integral representation. The strength of exact RG flows is that systematic approximations of the integrated flow correspond to systematic approximations to the full quantum theory. This property, in combination with the convergence behavior of the flow, is at the root of the predictive power of the formalism. The similarity of the different one-loop improved flows, including

\footnotetext{
*Email address: Daniel.Litim@cern.ch

†Email address: jmp@theorie3.physik.uni-erlangen.de
}

ERG flows, has fueled hopes that the scenario just described for exact flows may be valid in general.

Based on this picture, and prior to an application of a general one-loop improved flow to any physical problem, it is mandatory to either prove that a given flow is exact, or to unravel its inherent approximations. A way to settle these questions consists of a detailed comparison of one-loop improved flows with known exact flows. Within the derivative expansion, this has been studied in [1]. In this Rapid Communication, we take a different route and study one-loop improved RG equations within perturbation theory. It is shown that they only represent, in general, approximations to flows in the full theory. This result is achieved by a structural analysis of the flows, and by calculating the diagrammatic representation of the two-loop contributions to the effective action generated by the flow through an iterative formal integration. In general neither the graphs nor the combinatorial factors of the two-loop diagrams that originate from oneloop improved flows, are the correct ones. A full account of the present calculation together with a discussion of related issues will be presented in [7].

\section{ONE-LOOP IMPROVED RENORMALIZATION GROUP}

We briefly review the philosophy of a one-loop improved renormalization group. The starting point is the formal equation for the one-loop effective action:

$$
\Gamma^{1-\mathrm{loop}}=S_{c l}+\frac{1}{2} \operatorname{Tr} \ln S^{(2)} .
$$

The trace in Eq. (1) is ill-defined and requires-at least-an UV regularization. A one-loop improved RG is derived from Eq. (1) by first employing an explicit regularization, taking the derivative with respect to the cutoff scale $k$ and then substituting $S^{(2)}$ by $\Gamma^{(2)}$. Here, we concentrate on infrared regularizations; this does not make a difference for the flow itself, which in either case should be local in momentum space, e.g., only a small momentum range about $q^{2} \approx k^{2}$ contributes to the flow at fixed $k$.

Let us start with the derivation of the ERG flow [4-6]. Adding an infrared regulator $R$ (a momentum-dependent mass term) to $S^{(2)}$ in Eq. (1) and proceeding according to the one-loop improvement philosophy, we arrive at 


$$
\partial_{t} \Gamma_{k}=\frac{1}{2} \operatorname{Tr}\left(\Gamma_{k}^{(2)}+R\right)^{-1} \partial_{t} R,
$$

where $t=\ln k$ is the logarithmic infrared scale introduced via $R$. The regulator $R$ has to meet some requirements as a function of momentum and the cutoff scale, which are discussed at length in the literature. For our purpose these consistency requirements are irrelevant, since we only want to perform iterative formal integrations.

We emphasise that a general exact flow is the flow of some operator insertion within the theory. A first-principle derivation of the ERG, for example, is based on the insertion $\frac{1}{2} \int \phi R \phi$. Insisting on the one-loop nature of the flow, one is bound to an insertion which is at most quadratic in the fields. Otherwise, the corresponding exact flow would also contain higher-loop contributions. We conclude that an exact flow with a one-loop structure must depend linearly on the full propagator. This is indeed the case for the ERG flow (2).

Another possibility for regularizing the expression in Eq. (1) is to modify the trace itself by inserting an operator $\rho$ multiplicatively [8]. This amounts to the replacement $\operatorname{Tr} \ln S^{(2)} \rightarrow \operatorname{Tr} \rho \ln S^{(2)}$ in (1) and leads to the one-loop improved RG flow

$$
\partial_{t} \Gamma_{k}=\frac{1}{2} \operatorname{Tr} \partial_{t} \rho \ln \Gamma_{k}^{(2)} .
$$

The multiplicative structure of this flow is particularly convenient, when used in numerical applications. Note, that opposed to Eq. (2), the flow (3) depends on the logarithm of $\Gamma_{k}^{(2)}$. Based on this structure, we can already conclude that Eq. (3) cannot be exact.

Finally we consider a regularization based on a propertime representation of Eq. (1),

$$
\Gamma^{1 \text {-loop }}=S_{c l}-\frac{1}{2} \int \frac{d s}{s} \operatorname{Tr} \exp \left(-s S_{c l}^{(2)}\right) \text {. }
$$

Now we multiply the integrand in (4) by a regularizing function $f\left(s \Lambda^{2}\right)-f\left(s k^{2}\right)$ [9]. Proceeding along the lines of the one loop improvement we arrive at [2]

$$
\partial_{t} \Gamma_{k}=\frac{1}{2} \int_{0}^{\infty} \frac{d s}{s} \partial_{t} f \operatorname{Tr} \exp \left(-s \Gamma_{k}^{(2)}\right) .
$$

In order to facilitate the perturbative calculations below, we cast the flow equation (5) in a form which is more convenient for this purpose. This alternative representation also reveals more clearly the structure of the proper-time flows. To that end, we expand a general proper-time flow in the following basis set of regulator functions $f$ :

$$
\partial_{t} f(x ; m)=\frac{2}{\Gamma(m)} x^{m} \exp (-x) .
$$

Here, $x=k^{2} s$. Note that the IR behavior is controlled by the term $e^{-x}$, where $x$ serves as a mass. These flows cover all proper-time flows that have been studied in the literature [1-3,10-18]. Moreover, linear combinations $\Sigma_{m} d_{m} f(x ; m)$ of Eq. (6) with $\Sigma_{m} d_{m}=1$ cover all flows with masslike IR behavior. The trace in Eq. (5) can be written in terms of the normalized eigenfunctions $\Psi_{n}$ of $\Gamma_{k}^{(2)}$ with $\Gamma_{k}^{(2)} \Psi_{n}$ $=\lambda_{n} \Psi_{n}$. Within this representation we deal with simple $s$ integrals. By performing the $s$ integration we arrive at [7]

$$
\partial_{t} \Gamma_{k}=\operatorname{Tr}\left(\frac{k^{2}}{\Gamma_{k}^{(2)}+k^{2}}\right)^{m} .
$$

The operator kernel inside the trace is the $m$ th power of a Callan-Symanzik kernel. We note that the functional dependence of Eq. (7) on $\Gamma^{(2)}$ depends on the regularization. Above, we have argued that an exact one-loop flow has to depend linearly on the full propagator. Hence, Eq. (7) is not exact for $m \neq 1$ due to the nonlinear dependence of Eq. (7) on the full propagator.

In addition, Eq. (7) also signals that, at least in perturbation theory, the deviation of a general proper-time flow from an exact flow is regularization dependent. In contrast, for both Eqs. (2) and (3), the functional dependence on $\Gamma^{(2)}$ and, thus, the result of their formal integration is independent of the regularization. For Eq. (5), however, linear combinations of Eq. (7) span the space of all kernels which decay at least as $\left(\Gamma^{(2)}+k^{2}\right)^{-1}$ and reproduce the one-loop effective action. A general kernel trivially leads a to a nonunique end point of the flow. This result also implies that Eq. (5), in general, is not an exact flow.

\section{EFFECTIVE ACTION AT ONE LOOP}

Thus, prior to any use of the flows (3) and (5), it is mandatory to collect more information on their inherit deviation from exact flows. Here, this is done by explicitly calculating one-loop and two-loop effective actions following from the flows. This also serves as an independent proof of our general statements. We restrict ourselves to a scalar theory with one species of fields, but with general interaction. The results are easily generalized to arbitrary field content. As the flows (2), (3) and (5) are derived as one-loop improved flows from the one-loop effective action (1), their integrals reproduce the one-loop effective action in the limit, where the infrared cutoff tends to zero. It is instructive to see how this comes about. The one-loop contribution $\Delta \Gamma_{1}$ is given by

$$
\Delta \Gamma_{1}=\int_{\Lambda}^{k} \frac{d k^{\prime}}{k^{\prime}}\left(\partial_{t^{\prime}} \Gamma_{k^{\prime}}\right)_{1 \text {-loop }}
$$

Here, $\left(\partial_{t^{\prime}} \Gamma_{k^{\prime}}\right)_{1 \text {-loop }}$ stands for the right-hand sides in either of the flow equations (2), (3), or (5), with $\Gamma_{k}^{(2)}$ substituted by $S^{(2)}$. This is sufficient to obtain the effective action at one loop.

Consequently, integrating the ERG flow (2) leads to

$$
\Delta \Gamma_{1}=\frac{1}{2} \operatorname{Tr}\left[\ln \left(S^{(2)}+R\right)\right]_{\Lambda}^{k} .
$$

Note that even for $k \neq 0$ the expression functionally resembles the one-loop contribution to the effective action. Indeed, it is the UV regularized one-loop contribution for a theory with propagator $S^{(2)}+R$.

Integrating the one-loop improved flow (3) leads to 


$$
\Delta \Gamma_{1}=\frac{1}{2} \operatorname{Tr}\left[\rho \ln S^{(2)}\right]_{\Lambda}^{k} .
$$

Again this resembles the one-loop effective action for any $k$. In contrast to an ERG flow, however, it is impossible to interpret Eq. (10) as the one-loop contribution of an UVregularized modified theory.

Integrating the proper-time flow (7) at one loop, we get after a straightforward algebra

$$
\Delta \Gamma_{1}=\frac{1}{2 m} \operatorname{Tr}\left[\left(\frac{k^{\prime 2}}{S^{(2)}}\right)^{m}{ }_{2} F_{1}\left(m, m ; m+1 ;-\frac{k^{\prime 2}}{S^{(2)}}\right)\right]_{\Lambda}^{k},
$$

where ${ }_{p} F_{q}(x, y ; z ; w)$ is the generalized hypergeometric series. For integer $m$, the series in ${ }_{2} F_{1}$ in Eq. (11) can be summed up and there is a simpler representation for the oneloop contribution:

$$
\Delta \Gamma_{1}=\frac{1}{2} \operatorname{Tr}\left[\ln \left(S^{(2)}+k^{\prime 2}\right)-\sum_{n=1}^{m-1} \frac{1}{n}\left(\frac{k^{\prime 2}}{S^{(2)}+k^{\prime 2}}\right)^{n}\right]_{\Lambda}^{k} .
$$

For $k \neq 0$ Eq. (11) does not resemble the one-loop contribution to the effective action. Of course, for $k \rightarrow 0$, Eq. (11) reproduces the one-loop effective action $\frac{1}{2}\left[\operatorname{Tr} \ln \left(S^{(2)}+k^{2}\right)\right]_{\text {ren }}$ where the renormalization at $\Lambda$ is included.

\section{EFFECTIVE ACTION AT TWO LOOP}

As the ERG flow (2) has a first-principles derivation, obviously it has to reproduce the correct two-loop result. Structurally it belongs to the same class as the usual CallanSymanzik flow, and the calculation of diagrams and combinatorial prefactors of either flow goes along the same lines. Here, we only present the result of such a calculation. The two-loop contribution $\Delta \Gamma_{2}$ to the effective action obeying (2) is given by

$$
\begin{aligned}
\Delta \Gamma_{2}= & \int_{p p^{\prime} q q^{\prime}}\left[\frac{1}{8} G_{p p^{\prime}} S_{p^{\prime} p q q^{\prime}}^{(4)} G_{q^{\prime} q}\right. \\
& \left.-\frac{1}{12} \int_{l l^{\prime}} G_{p p^{\prime}} S_{p^{\prime} l q}^{(3)} G_{l l^{\prime}} S_{l^{\prime} p q^{\prime}}^{(3)} G_{q^{\prime} q}\right]_{\mathrm{ren} .},
\end{aligned}
$$

where the subscript "ren." indicates that these are renormalized diagrams due to the subtractions at $\Lambda$. We have introduced the abbreviations $G_{p p^{\prime}} \equiv\left(S^{(2)}+R\right)^{-1}\left(p, p^{\prime}\right)$, the vertices $S_{p_{1} \cdots p_{n}}^{(n)} \equiv \delta^{(n)} S / \delta \phi\left(p_{1}\right) \cdots \delta \phi\left(p_{n}\right)$, and a convenient short-hand notation for the momentum integrals $\int_{p_{1}} \cdots p_{n}$ $\equiv \int\left[d^{d} p_{1} /(2 \pi)^{d}\right] \cdots\left[d^{d} p_{n} /(2 \pi)^{d}\right]$. The combinatorial factors in Eq. (13) are in agreement with perturbation theory. Again, even for $k \neq 0$ the result (13) functionally resembles the perturbative structure. This analysis can be easily extended to any loop order. Note that one can always rewrite the integrands as total $t^{\prime}$ derivatives. Thus, the precise form of the regulator $R$ is irrelevant for the result, as it should.

Expanding the one-loop improved flow equation (3) at two loop leads to the following expression:

$$
\Delta \Gamma_{2}=\frac{1}{2} \int_{\Lambda}^{k} \frac{d k^{\prime}}{k^{\prime}} \int_{p p^{\prime} q q^{\prime}} \Delta \Gamma_{1, p p^{\prime}}^{(2)} G_{p^{\prime} q^{\prime}} \partial_{t^{\prime}} \rho_{q q^{\prime}}
$$

and $G=1 / S^{(2)}$. It is easy to rewrite the expression on the right-hand side of Eq. (14) as a total derivative, since the only $k$ dependence of $\Delta \Gamma_{1}^{(2)}$ is given by $\rho$. We finally get

$$
\begin{aligned}
\Delta \Gamma_{2}= & \int_{p p^{\prime} q q^{\prime}}\left[\frac{1}{8}(G \rho)_{p p^{\prime}} S_{p^{\prime} p q q^{\prime}}^{(4)}(G \rho)_{q^{\prime} q}\right. \\
& \left.-\frac{1}{8} \int_{l l^{\prime}}(G \rho)_{p p^{\prime}} S_{p^{\prime} l q}^{(3)} G_{l l^{\prime}} S_{l^{\prime} p q^{\prime}}^{(3)}(G \rho)_{q^{\prime} q}\right]_{\text {ren. }}
\end{aligned}
$$

Again, as for Eq. (13), the result does not depend on the regulator for $k=0$, where $\rho=1$. Differentiating Eq. (15) with respect to $k$ leads to the integrand of Eq. (14), as it should. The combinatorial factors of the diagrams in Eq. (15) do not match those in Eq. (13). Thus the flow (3) fails to reproduce perturbation theory beyond one loop.

Finally we discuss the proper-time flow (5). Below Eq. (7), we have already argued that the flow (5) is not an exact flow for a general regulator. Here, as an explicit example, we calculate the two loop effective action for $m=2$. Expanding the flow (5) at two loop we get

$$
\Delta \Gamma_{2}=-2 \int_{\Lambda}^{k} \frac{d k^{\prime}}{k^{\prime}} \int_{p p^{\prime}} \Delta \Gamma_{1, p p^{\prime}}^{(2)}\left(G k^{\prime 2} G k^{\prime 2} G\right)_{p^{\prime} p},
$$

where $G_{p p^{\prime}} \equiv\left(S^{(2)}+k^{\prime 2}\right)^{-1}\left(p, p^{\prime}\right)$. Note, that it is impossible to rewrite the integrand in Eq. (16) as a total derivative with respect to the scale parameter $t^{\prime}$. This already is a strong hint at the fact that one cannot get the correct twoloop result. Let us cast Eq. (16) in a form which shows explicitly how it deviates from perturbation theory. Using partial $t^{\prime}$ integrations we obtain, from Eq. (16), after some lengthy but straightforward algebra,

$$
\begin{aligned}
\Delta \Gamma_{2}= & \int_{p p^{\prime} q q^{\prime}}\left[\frac{1}{8} G_{p p^{\prime}} S_{p^{\prime} p q q^{\prime}}^{(4)} G_{q^{\prime} q}\right. \\
& \left.-\frac{1}{12} \int_{l l^{\prime}} G_{p p^{\prime}} S_{p^{\prime} l q}^{(3)} G_{l l^{\prime}} S_{l^{\prime} p q^{\prime}}^{(3)} G_{q^{\prime} q}\right]_{\mathrm{ren} .} \\
& -\frac{1}{2} \int_{\Lambda}^{k} \frac{d k^{\prime}}{k^{\prime}} \int_{p p^{\prime} q q^{\prime} l l^{\prime}}\left[\left(G k^{\prime 2} G\right)_{p p^{\prime}} S_{p^{\prime} q l}^{(3)}\right. \\
& \times\left(G k^{\prime 2} G\right)_{q q^{\prime}} S_{q^{\prime} p l^{\prime}}^{(3)}\left(G k^{\prime 2} G\right)_{\left.l^{\prime} l\right]} .
\end{aligned}
$$

Differentiating Eq. (17) with respect to $k$ leads to the integrand of Eq. (16), as it should. The first two terms in Eq. (17) correspond to the correct two-loop result as presented in Eq. (13). The last term denotes the deviation from standard perturbation theory. The $d \ln k^{\prime}$ integrand of the last term in Eq. (17) is the nonstandard diagram depicted in Fig. 1. The last term on the right-hand side of Eq. (17) cannot be absorbed in renormalization constants. It contains arbitrary powers in fields and momenta and does not integrate to zero in the limit $k \rightarrow 0$ and $\Lambda \rightarrow \infty$. For massive theories both limits are safe. 


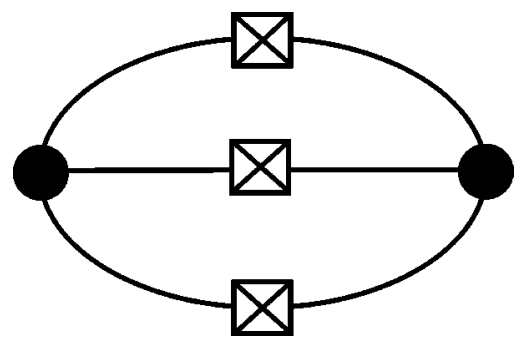

FIG. 1. The integrand of the nonstandard term in Eq. (17). The two vertices $S^{(3)}$ are denoted by $\boldsymbol{O}$, the six internal lines are the propagators $G=\left(S^{(2)}+k^{\prime 2}\right)^{-1}$, and the three insertions correspond to $k^{\prime 2}$.

Consequently this term displays a nontrivial deviation of the proper-time flow from perturbation theory. The form of the integrand is that of the sunset graph where all propagators have been substituted by their squares. This is clearly related to the fact that the form of the proper-time flow is that of a Callan-Symanzik flow with all propagators substituted by their squares.

To be more explicit, consider the example of a massive $\phi^{4}$ theory with mass $M$ and quartic interaction $\left(\lambda / 4\right.$ ! ) $\int d^{d} x \phi^{4}$. The contribution of the nonstandard diagram to the propagator is obtained after taking the second derivative with respect to the fields in Eq. (17) at $\phi=0$. We find

$$
\begin{gathered}
\lambda^{2} \int_{\infty}^{0} \frac{d k}{k} \int \frac{d^{d} q}{(2 \pi)^{d}} \frac{d^{d} l}{(2 \pi)^{d}}\left[\frac{k^{2}}{\left(k^{2}+M^{2}+q^{2}\right)^{2}}\right. \\
\left.\quad \times \frac{k^{2}}{\left(k^{2}+M^{2}+l^{2}\right)^{2}} \frac{k^{2}}{\left(k^{2}+M^{2}+(l+q-p)^{2}\right)^{2}}\right] .
\end{gathered}
$$

The integrand is strictly positive. Hence the integral is nonvanishing. Moreover, it has a nontrivial momentum dependence. This can be seen by evaluating the limits $p \rightarrow 0$ and $p \rightarrow \infty$. For $p \rightarrow 0$ we are left with a nonvanishing constant. In turn, for $p \rightarrow \infty$ the expression in Eq. (18) vanishes.

\section{DISCUSSION}

Having established that neither (3) nor, in general, (5) provides exact flows, we want to understand what precisely causes the deviation from perturbation theory. First we recall the argument made prior to Eq. (3): A general exact flow is related to the flow of an operator insertion in the theory. Demanding, additionally, that the flow has a one-loop structure restricts possible insertions to operators quadratic in the fields. Consequently such a flow has to depend linearly on the full propagator.

For a general flow it might be hard to decide whether one has such a situation. Already for general proper-time flows we had to take the detour of expanding general flows in the basis (6) in order to reach a conclusion. Thus, we would like to provide an additional criterion, which also reflects the necessity of a linear dependence on the full propagator. Indeed, a sufficient condition for a RG equation to reproduce perturbation theory can be deduced from the iterative structure of the perturbation series: It suffices that the solution of a RG equation has the same iterative structure even at nonvanishing cutoff. Without this property, the corresponding RG equation has to satisfy an infinite tower of iterative constraints in order to reproduce perturbation theory in the limit, where the infrared cutoff tends to zero. Consequently, one can assess from the structure of the one-loop effective action at $k \neq 0$ whether a flow is likely to reproduce perturbation theory.

The iterative structure discussed above is absent in the one-loop effective action given in Eq. (11) for $k \neq 0$. Moreover, it cannot be regained by considering linear combinations of regulators (6). Despite this discouraging fact, let us shed some more light on the structure of proper-time flows. It is not possible to integrate a general proper-time flow beyond one loop without knowing the precise form of the regulator. Still, there are recursive relations between different proper-time flows at a given loop order. These relations tell us how the flows differ from each other for arbitrary $m$, integer or not. At two loop, and with $G=\left(S^{(2)}+k^{2}\right)^{-1}$, the most general recursion relation is given by [7]

$$
\begin{aligned}
\Delta \Gamma_{2, m}-\Delta \Gamma_{2, m-1}= & \frac{1}{2} \int_{\infty}^{0} \frac{d k}{k} \operatorname{Tr}\left[\left(G k^{2}\right)^{m-1} G\right. \\
& \times\left(\frac{m}{m-1} k^{2} G-1\right) \frac{\delta^{2}}{(\delta \phi)^{2}} \\
& \left.\times \operatorname{Tr}\left(G k^{2}\right)^{m-1}\right],
\end{aligned}
$$

apart from irrelevant terms from the different renormalization procedures for the two flows. The difference (19) (or, more generally, $\Delta \Gamma_{2, m}-\Delta \Gamma_{2, m-n}$ with integer $n$ ) depends on arbitrarily high powers of the fields and does not integrate to zero.

Equation (19) can be used to give an independent explicit proof of the nonexactness of general proper-time flows. To that end, let us assume for a moment that the proper-time flow for a particular $m_{0}$ is exact. Then it follows from Eq. (19) that all flows with $m=m_{0}+n$ for integer $n$ are not exact, because the corresponding terms (19) do not vanish identically in the fields. Hence, of all proper-time flows of the form (5) with regulators (6) or finite linear combination thereof, the set of exact flows is of measure zero. This has an immediate consequence for flows with integer $m$. The Callan-Symanzik flow $(m=1)$ is exact, but any flow with integer $m>1$, or any linear combinations thereof, are not exact. Hence, the structure of the findings for $m=2$ is present for arbitrary $m$, and Eq. (19) provides an independent explicit proof for the general statement derived after Eq. (7).

Thus, for proper-time flows, we arrive at the following picture. The only known exact proper-time flow is the Callan-Symanzik flow. Other exact proper-time flows-if they exist-would require a linear dependence on the full propagator, possibly in some disguise. Based on our findings, no further exact flows can be found within the set of regulators (6), which covers all flows previously studied in the literature. Of course, it is not excluded that a regulator, 
which is represented by an infinite series of regulators (6), is exact. However, there is no a priori criterion upon which one could embark and construct such a regulator.

To summarize, we have shown that the one-loop improved flows (3) and, in general, (5) are not exact flows. We have shown explicitly, that they fail at the first nontrivial order, at two loop. These results imply that hopes expressed in the literature-suggesting that the RG flows (3) and (5) correspond to exact flows only with a different implementation of the regularization-cannot be maintained. In fact, these flows are substantially different from exact flows, and describe at best approximations to the latter. Justification of their use requires a deep understanding of the inherent ap-

[1] D. F. Litim and J. M. Pawlowski, Phys. Lett. B 516, 197 (2001).

[2] S. B. Liao, Phys. Rev. D 53, 2020 (1996).

[3] S. B. Liao, Phys. Rev. D 56, 5008 (1997).

[4] J. Polchinski, Nucl. Phys. B231, 269 (1984).

[5] C. Wetterich, Phys. Lett. B 301, 90 (1993); U. Ellwanger, Z. Phys. C 62, 503 (1994); T. R. Morris, Int. J. Mod. Phys. A 9, 2411 (1994).

[6] C. Bagnuls and C. Bervillier, Phys. Rep. 348, 91 (2001); D. F. Litim and J. M. Pawlowski, hep-th/9901063; J. Berges, N. Tetradis, and C. Wetterich, hep-ph/0005122.

[7] D. F. Litim and J. M. Pawlowski, hep-th/0202188.

[8] S. B. Liao, C. Y. Lin, and M. Strickland, hep-th/0010100.

[9] M. Oleszczuk, Z. Phys. C 64, 533 (1994). proximation in order to furnish these methods with predictive power. This question has only been addressed within the derivative expansion [1]. However, the potential benefits of general one-loop improved RG flows within numerical implementations justify further investigations. An extensive study of this problem, including a more detailed account of the present calculations, will be given elsewhere [7].

\section{ACKNOWLEDGMENTS}

J.M.P. thanks CERN for hospitality. D.F.L. has been supported under EC contract no. HPMF-CT-1999-00404.

[10] R. Floreanini and R. Percacci, Phys. Lett. B 356, 205 (1995).

[11] B. J. Schäfer and H. Pirner, Nucl. Phys. A660, 439 (1999).

[12] J. Meyer, G. Papp, H. J. Pirner, and T. Kunihiro, Phys. Rev. C 61, 035202 (2000).

[13] G. Papp, B. J. Schäfer, H. J. Pirner, and J. Wambach, Phys. Rev. D 61, 096002 (2000).

[14] O. Bohr, B. J. Schäfer, and J. Wambach, Int. J. Mod. Phys. A 16, 3823 (2001).

[15] J. Meyer, K. Schwenzer, H. J. Pirner, and A. Deandrea, Phys. Lett. B 526, 79 (2002).

[16] A. Bonanno and D. Zappalà, Phys. Lett. B 504, 181 (2001).

[17] M. Mazza and D. Zappalà, Phys. Rev. D 64, 105013 (2001).

[18] D. Zappalà, Phys. Lett. A 290, 35 (2001). 